\title{
Reviews
}

\section{Brand vandals: Reputation wreckers and how to build better defences}

\author{
Steve Earl and Stephen Waddington \\ Bloomsbury Publishing, London, 2013; softback; £12.99; 271pp; \\ ISBN: 978-1472905208
}

Journal of Direct, Data and Digital Marketing Practice (2014) 15, 352-354. doi:10.1057/dddmp.2014.14

\section{Nature of the threat is changing}

\section{Social media 'terrorists'}

\section{Becoming fit-for-purpose}

The thesis of this book is that the reputations of brands are threatened by forms of attack that are different from what they used to be. Organisations need to be aware of these changes and redesign their corporate communications teams accordingly.

When print and broadcast media were the dominant communications channels, brands could be defended against organised attack relatively easily, since the information about a brand that consumers were fed was channelled through a relatively small number of titles or stations. The gatekeepers controlling these channels' content were easy to identify, communicate with and influence.

Opportunities for communication occurred at set times during the day. Stories were selected on the basis of predictable criteria. They unfolded at a pace that allowed time for corporate chiefs to meet, discuss and decide their next moves.

By contrast, social media is characterised by opponents who are ubiquitous, operate in real time and, because they are anonymous, can be engaged with only in open dialogue. If war is an appropriate metaphor, dealing with 'terrorists' requires a different strategy from dealing with traditional 'ground troops'.

The authors of 'Brand Vandals' are highly experienced and successful evangelists on this new form of 'warfare'. In Chapters 1-6, they alert corporate chieftains to the shape and seriousness of the emerging threat. Then in Chapters 7-12 they set out strategies for developing an effective defence capability. In particular, they explain the mode of operation of the communications team in the age of social media, the new skill sets that it demands and the changes to the command structure needed to make it effective.

What, then, are the qualities required of a fit-for-purpose communications team in the age of social media? Here are some of those advocated:

- Flexibility - Centralised command and control is unlikely to be effective where the source and the nature of the attack are difficult to 
Taking responsibility for decisions

\section{So what is 'vandalism'?}

\section{Do these strategies work for all brands?}

predict in advance and where social media, operating in real time and across a very wide front, demands an instant response.

- Preparedness - Because communications decisions need to be taken in real time and by each member of the communications team, members need greater training in communication strategies that will effectively counter social media attacks.

- Awareness - Because the sources of reputational attack are less predictable, team members need to watch and listen more intently, scanning social media for potential brand wreckers before they have the chance to build momentum.

- Dialogue - The democratic nature of social media requires a much stronger level of interactive audience engagement than the set-piece radio interviews and media releases of the pre-social media era.

- Transparency - The democratisation of media content increases the risk of uncomfortable facts being revealed. Cover-up becomes a more risky strategy.

- Authenticity - Employees and partners should be actively involved in the defence strategy since audiences are influenced by the accounts of people who they consider to be disinterested.

- Humour - To be effective, social media content has to engage. Humour is an effective means of contextualising and reversing opponents' arguments.

Putting all this into practice does demand a smarter and more multifaceted communications team whose members are steady under fire and have the courage to take personal responsibility for their own decisions.

To a large degree these truths are self-evident. The authors accept this. But they are almost certainly justified in arguing that more work needs to be done to embed these principles in the practice of legacy structures. After all, they do run counter to the drive of most large organisations to reduce uncertainty wherever possible through the use of ever more sophisticated management controls.

While these prescriptions are undoubtedly relevant to different types of negative content, the reader would benefit from clarification of what is deemed to constitute 'vandalism'. Are we here describing the occasional antics of a hard core, happy to seize any opportunity for using sophisticated social media techniques to trash what they perceive as exploitative and over-powerful brands?

Or should communications teams be engaged in continuous defence against the much larger number who use social media to communicate low-level grievances that they would otherwise have complained about to friends and colleagues face to face? If so, how can the communications team understand operational processes well enough to distinguish destructive comments from legitimate calls for particular improvements where business processes are poorly designed?

Likewise, would it be surprising if strategies for defending brands with which users have a strong emotional involvement (such as Burberry, BMW) were equally effective for brands (such as Tesco, Barclays) that 


\section{Enabling opponents to self-organise}

consumers select largely on the basis of function and convenience, but subject to the brand satisfying socially accepted standards of appropriate corporate conduct?

As a comparatively light user of social media, I could not help wondering how pervasive the effect of social media is on brand reputation. What proportion of the adult population are infrequent or non-users of social media? Of those who are regular users, how many are disposed to engage in conversations about brands?

Or does social media's impact on brand reputation depend not so much on its reach as on it being a medium whereby opinion can rapidly permeate the awareness of an elite of highly influential communicators? Or is it because it is such an effective mobilisation tool, enabling opponents to self-organise rapidly and effectively?

For advocates struggling to influence sceptical colleagues, this will prove a highly motivating text with no shortage of detailed advice and plenty of relevant examples.

Richard Webber

\section{Data smart: Using data science to transform information into insight}

Journal of Direct, Data and Digital Marketing Practice (2014) 15, 354-355. doi:10.1057/dddmp.2014.33

Real value for self-help

Highest possible

Bayes and a bag of
words
What do you expect when you read a business or reference book? Ideally, the knowledge it contains should transfer positively into your working life as soon as possible. After all, why bother if there is no hope of that happening? (Although the continued growth of the self-help publishing sector suggests that hope tends to triumph over experience.) When that knowledge transfer actually happens, the book in question becomes one you treasure, recommend and constantly return to.

That is why I have to give John W. Foreman's new book (Wiley, US, ISBN: 978-1118661468) the highest possible recommendation because of my direct experience of what it contains transferring into my professional life. In April, the IDM ran its Data Discovery Workshop to provide a group of students an insight into how data is used in the commercial world and encourage these mathematicians, psychologists, engineers and the like to consider a career in the industry.

A drinks reception was thrown for them on the evening of the second day. Earlier, I had some time to kill and so I continued to read my review copy of Data Smart. In Chapter 3, called 'Naïve Bayes and the incredible lightness of being an idiot', I learned about techniques for classifying texts (such as tweets) to understand whether they are positive or negative. Based 